\title{
Ửng dụng mô hình chấp nhận và sử dụng công nghệ hợp nhất (UTAUT): Trường họ̣p sử dụng phần mềm quản lý văn bản của viên chức trường Đại học Quốc tế
}

\section{An application of Unified Technology Acceptance Use of Technology (UTAUT): The case of Portal Office system by officials of the International University}

\author{
Nguyễn Ngọc Duy Phương ${ }^{2}$, Huỳnh Vĩnh Trường ${ }^{1}$ \\ ${ }^{1}$ Trường Đại học Quốc tế, ĐHQG-HCM, Việt Nam \\ *Tác giả liên hệ, Email: nndphuong@hcmiu.edu.vn
}

THÔNG TIN

DOI: $10.46223 / \mathrm{HCMCOUJS.}$

econ.vi.17.3.1966.2022

Ngày nhận: 26/06/2021

Ngày nhận lại: 09/07/2021

Duyệt đăng: 10/07/2021

Tù khóa:

chính phủ điện tử; hành vi sử dụng; UTAUT; ý định hành vi

Keywords:

E-goverment; use behavior; UTAUT; behavioral intention

\section{TÓM TẮT}

Úng dụng công nghệ thông tin trong quản lý hành chính nhà nước là một trong những chủ trương mang tính cấp thiết, đáp ứng nhu cầu phát triển của xã hội, đặc biệt là trong tình hình đại dịch Covid-19 diễn biến phức tạp. Cán bộ - viên chức cần được liên tục trang bị kiến thức, kỹ năng và các trang thiết bị để đáp ứng những yêu cầu ngày càng cao trong giải quyết công việc. Nghiên cứu này xác định khả năng ứng dụng của mô hình UTAUT (Venkatesh, Morris, Davis, \& Davis, 2003) với sự hỗ trợ của mô hình TAM nhằm mục đích tìm hiểu những yếu tố ảnh hưởng đến ý định hành vi của viên chức đối với việc xử lý các tác vụ thông qua ứng dụng phần mềm Portal Office thuộc về lĩnh vực môi trường giáo dục nằm trong khu vực công. Một cuộc khảo sát 100 viên chức được thực hiện với cách tiếp cận bằng phương pháp nghiên cứu định lượng. Kết quả chỉ ra rằng Nhận thức sự hữu ích, Nhận thức dễ sử dụng, Thói quen và Các điều kiện thuận lợi đều có ảnh hưởng đến ý định và hành vi sử dụng hệ thống Portal Office. Về hàm ý quản trị, kết quả cho thấy rằng Ban lãnh đạo cần phải xây dựng thói quen sử dụng, tuyên truyền về lợi ích, tính thân thiện của phần mềm và đáp ứng các điều kiện thuận tiện hỗ trợ cho việc sử dụng phần mềm, từ đó thúc đẩy năng suất làm việc hiệu quả của viên chức.

\section{ABSTRACT}

Applying information technology in state administrative management is one of the urgent policies to meet social development needs, especially in the turbulent situation of the Covid-19 pandemic. Cadres - public employees need to be continuously equipped with knowledge, skills, and equipment to improve work efficiency. This study determines the applicability of the UTAUT (Venkatesh, Morris, Davis, \& Davis, 2003) with the support of TAM model to examine what factors affect the behavioral intentions of employees towards using Portal Office 
software for work in the context of public higher education. The study employs a quantitative method by a self-administered survey of 100 officials. The results show that perceived usefulness, ease of use, habits, and facilitation conditions affect behavioral intention and actual use of the Portal Office system. In terms of governance implications, the findings suggest that the Board of Directors may need to build usage habits, propagate the benefits and friendliness of the software, and meet convenient conditions that support the use of the software, thereby promoting effective work productivity of employees.

\section{Giới thiệu}

Trong những năm qua, trước sự phát triển mạnh mẽ của cách mạng công nghiệp 4.0 (Trí tuệ nhân tạo, Internet vạn vật, Dữ liệu lớn), ứng dụng công nghệ thông tin được xác định là công cụ hữu hiệu trong việc cải cách hành chính. Theo báo cáo số 6466/BC-BNV ngày 24/12/2019 của Bộ Nội vụ về tình hình thực hiện công tác cải cách hành chính năm 2019 về đầu tư, nâng cấp, sử dụng phần mềm quản lý văn bản và điều hành: 63/95 cơ quan Trung ương và địa phương đã nâng cấp với các tính năng mới và đã khởi chạy phần mềm quản lý văn bản và điều hành, 28 cơ quan đang thực hiện nâng cấp. Gần như tất cả thủ trưởng các cơ quan, đơn vị thuộc bộ, cơ quan ngang bộ đều sử dụng phần mềm quản lý văn bản và điều hành để giải quyết, điều hành công việc một cách thường xuyên (Bộ Nội vụ, 2019). Việc ứng dụng giải quyết hồ sơ trên nền điện tử đã được Văn phòng chính phủ triển khai từ nhiều năm nay với mục tiêu: ứng dụng công nghệ thông tin, hướng đến "Văn phòng chính phủ phi giấy tờ". Tại phiên họp của Ủy ban Quốc gia về Chính phủ điện tử ngày 10/03/2021, Bộ trưởng Thông tin và Truyền thông Nguyễn Mạnh Hùng cho biết dự kiến sẽ cơ bản hoàn thành Chính phủ điện tử vào năm 2021 với chỉ tiêu $100 \%$ dịch vụ công trực tuyến mức độ 04 , bên cạnh đó, năm 2025 sẽ hình thành Chính phủ số (Gia Huy, 2021). Quản lý hành chính nhà nước đi kèm với ứng dụng công nghệ là chủ trương lớn của Đảng và Nhà nước, phù hợp với quy luật phát triển xã hội, đã được triển khai, giúp cán bộ công chức có bước nhận thức cơ bản về sự cần thiết của việc đẩy mạnh ứng dụng công nghệ thông tin vào công tác quản lý hành chính nhà nước. Bên cạnh đó, trong bối cảnh dịch bệnh Covid-19 (một đại dịch bệnh truyền nhiễm với tác nhân là virus Corona gây hội chứng hô hấp cấp tính nặng) đang gây ra những ảnh hưởng lớn đến đời sống xã hội trên phạm vi toàn cầu từ đầu năm 2020, người dân, doanh nghiệp, các bộ công chức, viên chức, ... được khuyến khích tăng cường sử dụng dịch vụ công trực tuyến (Bộ Y tế, 2020), việc đẩy mạnh và phát triển ứng dụng công nghệ thông tin trong tất cả các hoạt động hành chính công được khuyến nghị và đang ngày càng trở nên bức thiết hơn. Tăng cường ứng dụng công nghệ thông tin trong quản lý đào tạo tại các trường đại học là cần thiết, góp phần nâng cao chất lượng và hiệu quả của công tác quản lý đào tạo, bồi dưỡng cán bộ, xây dựng các cơ sở đại học. Nhiều nghiên cứu về việc chấp nhận và sử dụng công nghệ bằng mô hình UTAUT đã và đang thực sự được tiến hành trong khu vực công và các cơ sở giáo dục đại học trên toàn thế giới với các tác giả Raman và Don (2013), Teo (2011), Wong, Teo, và Russo (2013). Tuy nhiên, có rất ít bài báo nghiên cứu được đăng trên các ấn phẩm có uy tín về việc sử dụng mô hình chấp nhận ứng dụng sử dụng công nghệ trong các cơ sở giáo dục đại học ở Việt Nam (Nguyen, Nguyen, \& Cao, 2014; Pham, Dang, \& Le, 2020). Nghiên cứu này tập trung vào sự ảnh hưởng giữa niềm tin cải thiện năng suất, nhận thức về sự dễ dàng sử dụng công nghệ mới, thói quen và các điều kiện thuận tiện hỗ trợ sử dụng phần mềm hệ thống đến dự định và từ đó tác động đến hành vi sử dụng của viên chức trường Đại học Quốc tế (ĐHQG-HCM). Portal Office hay Văn 
phòng điện tử là một phần mềm quản lý dựa trên nền tảng Web được phát triển bởi Công ty TNHH Giải pháp Phần mềm Việt (Vietnam Software Solutions Co., ltd). Chức năng chính của phần mềm bao gồm: quản lý, phân loại các hình thức văn bản đến, đi, nội bộ trên hệ thống; xây dựng các tập tài liệu lưu trữ online; cấp quyền kiểm soát quá trình xử lý văn bản từ giai đoạn tiếp nhận cho đến khi hoàn thành. Bên cạnh đó, phần mềm có khả năng xử lý chữ ký số, đọc và định dạng của Microsoft Office và liên thông văn bản quốc gia.

\section{Cơ sở lý thuyết và mô hình nghiên cứu}

\subsection{Khái niệm Hành vi người tiêu dùng, ý định hành vi, và chấp nhận sử dụng công nghệ thông tin}

Trong phạm vi bài nghiên cứu này, khái niệm Hành vi được biết đến cụ thể là "Hành vi người tiêu dùng" được xem như những phản ứng cảm xúc, nhận thức hoặc các phản ứng có thể quan sát được trước những tác động xuyên suốt liên quan đến việc mua và xử lý hàng hóa, dịch vụ của người tiêu dùng (Kardes \& Cronley, 2015). Hành vi người tiêu dùng có thể được định nghĩa là quá trình ra quyết định và hoạt động thể chất, hành động mua, đánh giá, sử dụng và thải bỏ các sản phẩm và dịch vụ đều là một phần của quá trình này. Ở cấp độ vi mô, nó liên quan đến việc hiểu người tiêu dùng nhằm mục đích giúp một công ty hoặc tổ chức đạt được các mục tiêu của mình (Khan, 2007).

Warshaw và Davis (1985) xác định rằng Ý định hành vi giống như mức độ sắp xếp một tập hợp những ý định theo một trình tự nhất định mà một người lập ra một cách sáng suốt để thực hiện hoặc không một số hành vi cụ thể trong tương lai được chỉ định trước. Davis, Bagozzi, và Warshaw (1989) nhận thấy rằng Ý định hành vi sử dụng hệ thống có tương đồng lớn với việc sử dụng. Bên cạnh đó, Ý định hành vi là đặc điểm cấu thành chính quyết định hành vi người dùng trong khi các yếu tố khác gián tiếp tác động đến hành vi của họ thông qua Ý định hành vi. Hill, Smith, và Mann (1987) cũng chỉ ra rằng thông qua Ý định hành vi có thể dự đoán được hành động một cách đáng kể. Tóm lại, Ý định hành vi có thể xem như mức cơ sở so sánh mà một cá nhân có nhận thức, sẵn sàng lập kế hoạch để đưa ra quyết định có hay không việc thực hiện hành vi.

Nghiên cứu của Kim và Kankanhalli (2009), Lippert và Davis (2006), và Sharma (2013) cho rằng khi giới thiệu các công nghệ mới, việc chấp nhận thay đổi bắt đầu từ trong chính mỗi cá nhân và điều này có thể bị ảnh hưởng bởi cách họ cảm nhận các ứng dụng mới sẽ ảnh hưởng đến hiệu suất công việc của họ như thế nào. Hidayanto và Ekawati (2010) đã kết luận rằng việc chấp nhận thay đổi có thành công hay không sẽ phụ thuộc vào sự chấp nhận của người dùng và sử dụng công nghệ mới của tổ chức. Mức độ chấp nhận của người dùng là một yếu tố quan trọng cần xem xét vì nó quyết định việc áp dụng, cài đặt và sử dụng công nghệ thông tin nơi làm việc (Agarwal \& Karahanna, 2000; Lippert \& Davis, 2006).

\subsection{Các lý thuyết liên quan}

\subsubsection{Mô hình Chấp nhận Công nghệ (TAM)}

Trong mô hình Chấp nhận Công nghệ (TAM), yếu tố Nhận thức dễ sử dụng có trực tiếp tác động đến biến Nhận thức sự hữu ích. Davis (1985) xác định khái niệm Nhận thức sự hữu ích là mức độ mà một cá nhân tin rằng sử dụng một hệ thống cụ thể sẽ nâng cao hiệu suất công việc của người đó; Nhận thức dễ sử dụng là mức độ mà một cá nhân tin rằng việc sử dụng một hệ thống cụ thể sẽ không cần nỗ lực về thể chất và tinh thần. Nhận thức sự hữu ích và Nhận thức dễ sử dụng đều được nhận thấy là có trực tiếp ảnh hưởng đến Ý định hành vi. 


\subsubsection{Mô hình Chấp nhận và sư dụng công nghệ hợp nhất (UTAUT)}

Venkatesh và cộng sự (2003) đã phát triển mô hình Chấp nhận và sử dụng công nghệ hợp nhất (UTAUT) và đã xác định các yếu tố quyết định như Nỗ lực kỳ vọng, Hiệu suất mong đợi, Ảnh hưởng xã hội, Các điều kiện thuận lợi và các nhân tố điều tiết như Giới tính, Tuổi tác, Trải nghiệm, Sự tự nguyện sử dụng. Trong đó, Nỗ lực kỳ vọng được định nghĩa là mức độ dễ dàng liên quan đến việc sử dụng hệ thống; Hiệu suất mong đợi được định nghĩa là mức độ mà một cá nhân tin rằng việc sử dụng các hệ thống mới sẽ giúp cho người đó đạt được năng suất trong công việc; Ảnh hưởng xã hội được xem là mức độ mà một cá nhân cảm thấy tầm quan trọng của việc bị ảnh hưởng bởi ý tưởng của những người xung quanh rằng họ nên sử dụng hệ thống mới; Điều kiện thuận lợi được định nghĩa là mức độ mà một cá nhân có niềm tin rằng cơ sở hạ tầng kỹ thuật của tổ chức đủ điều kiện để hỗ trợ cho hệ thống (Venkatesh \& ctg., 2003). UTAUT là một mô hình kết hợp một số mô hình trước đó về sự chấp nhận của người dùng đối với các hệ thống mới, bao gồm mô hình TAM. Từ góc độ lý thuyết, UTAUT cung cấp một cái nhìn về cách các yếu tố ảnh hưởng đến Ý định và Hành vi phát triển theo thời gian. UTAUT đã được thử nghiệm và cho thấy hiệu quả tốt hơn so với các mô hình cạnh tranh khác (Venkatesh \& ctg., 2003; Venkatesh \& Zhang, 2010).

\subsubsection{Mô hình Mở rộng chấp nhận và sủ dụng công nghệ hợp nhất - UTAUT2}

UTAUT2 được đề xuất như là một mô hình hữu ích để hiểu việc sử dụng công nghệ của người tiêu dùng nói chung. Trong mô hình này, cá nhân có đặc điểm là để Thói quen tác động đến Ý định hành vi. Venkatesh, Thong, và Xu (2012) xem Thói quen là một cấu trúc tri giác phản ánh kết quả của những trải nghiệm trước đó. UTAUT2 mô hình hóa cách Thói quen ảnh hưởng trực tiếp và gián tiếp đến Hành vi sử dụng thông qua Ý định hành vi Các bổ sung được đề xuất cho UTAUT2, theo Venkatesh và cộng sự (2012), phản ánh những sửa đổi lớn các yếu tố giải thích ý định hành vi và sử dụng công nghệ.

\subsection{Giả thuyết và mô hình nghiên cứu}

\subsubsection{Mối quan hệ giũa Nhận thức sụ hưu ích, Nhận thức dễ sử dụng và Ý định hành vi}

Davis và cộng sự (1989) cũng báo cáo rằng Nhận thức dễ sử dụng đã được phát hiện là có sự ảnh hưởng lớn liên quan đến Ý định và hành vi sử dụng. Các báo cáo trong các nghiên cứu của Adams, Nelson, và Todd (1992); Agarwal và Karahanna (2000); Davis và cộng sự (1989); Doll, Hendrickson, và Deng (1998); Segars và Grover (1993) đã xác minh rằng Nhận thức sự hữu ích và tính Nhận thức dễ sử dụng là yếu tố then chốt trong cấu trúc sự chấp nhận của cá nhân. Tuy nhiên, hai yếu tố này có thể không thể hiện đúng sự chấp nhận của người dùng Internet Banking (Hsu \& Lu, 2004). Để kiểm chứng các lập luận trên, tác giả đề xuất giả thuyết:

H1: Nhận thức sụ hưu ích sẽ có ảnh hưởng trục tiếp đến Ý định sủ dụng phần mềm Portal Office cuia viên chúc

H2: Nhận thức dễ sư dụng sẽ có ảnh hưởng trục tiếp đến Ý định hành vi sủ dụng phần mềm Portal Office của viên chức

\subsubsection{Mối quan hệ giữa Nhận thức dễ sử dụng và Nhận thức sụ hữu ích}

Nhận thức dễ sử dụng không ngừng ảnh hưởng trực tiếp hoặc gián tiếp đến Ý định hành vi thông qua Nhận thức sự hữu ích (Agarwal \& Karahanna, 2000; Hsu \& Lu, 2004). Một số lập luận trong những nghiên cứu trước đây khẳng định rằng có một mối quan hệ tích cực giữa Nhận thức sự hữu ích và Nhận thức dễ sử dụng khi chấp nhận công nghệ thông tin (Gefen \& Straub, 2000; Venkatesh \& Davis, 2000) và phát hiện này cũng đã được xác nhận trong sử dụng công nghệ Internet (Lederer, Maupin, Sena, \& Zhuang, 2000; Moon \& Kim, 2001). Theo đó, tác giả đề xuất giả thuyết: 
H3: Nhận thức dễ sử dụng có mối quan hệ tích cực với Nhận thức sụ hữu ích phần mềm Portal Office

\subsubsection{Mối quan hệ giũa Thói quen đến Ý định sủ dụng và Hành vi sủ dụng}

Thói quen đã được chứng minh là một yếu tố quan trọng dự đoán việc sử dụng công nghệ (Kim \& Malhotra, 2005; Kim, Malhotra, \& Narasimhan, 2005; Limayem, Hirt, \& Cheung, 2007). Nghiên cứu của Pham và cộng sự (2020) cho thấy Thói quen của giáo viên có tác động đến hành vi sử dụng công nghệ thông tin trong bài giảng của giáo viên. Theo Arenas, Peral, và Ramón (2015), Thói quen là một trong những yếu tố chính tạo nên và giải thích Hành vi sử dụng trực tiếp và gián tiếp thông qua Ý định hành vi. Tuy nhiên, theo Nguyen và cộng sự (2014), Thói quen có tác động không đáng kể đến Ý định hành vi sử dụng công nghệ. Raman và Don (2013) chỉ ra rằng Thói quen không có tác động tích cực đến Ý định hành vi hoặc Hành vi sử dụng. Để kiểm chứng mối quan hệ này, tác giả đưa ra giải thuyết:

H4: Thói quen sẽ có ảnh hương trục tiếp đến Ý định sư dụng phần mềm Portal Office của viên chưc

H5: Thói quen sẽ có ảnh hương trục tiếp đến Hành vi sủ dụng phần mềm Portal Office của viên chức hệ thống

2.3.4. Mối quan hệ giũa Các điều kiện thuận lợi đến Ý định sủ dụng và Hành vi sử dụng

Kết quả nghiên cứu của Alalwan, Dwivedi, Rana, Lal, và Williams (2015) cho thấy Các điều kiện thuận lợi có thể tác động trực tiếp đến việc dùng máy tính và hệ thống trên thực tế. Teo (2011) chỉ ra rằng Điều kiện thuận lợi có ảnh hưởng trực tiếp đến Hành vi. Các điều kiện thuận lợi cho một công nghệ có mối tương quan tích cực với việc sử dụng công nghệ. Theo Im, Hong, và Kang (2011), nếu có nhiều điều kiện thuận lợi hỗ trợ thì mọi người sẽ có nhiều khả năng hơn để áp dụng công nghệ đó. Nguyen và cộng sự (2014) kết luận rằng Điều kiện thuận lợi ảnh hưởng không đáng kể đến Hành vi sử dụng E-learning dựa trên điện toán đám mây. Do đó, tác giả đưa ra giả thuyết:

H6: Điều kiện thuận lợi sẽ có ảnh huởng trục tiếp đến Hành vi sủ dụng phần mềm Portal Office của viên chúc

\subsubsection{Mối quan hệ giũa Ý định hành vi đến Hành vi sư dụng hệ thống}

Venkatesh và cộng sự (2003) công nhận rằng Ý định hành vi là một yếu tố dự báo quan trọng của Hành vi sử dụng công nghệ. Wang (2016) kết luận rằng Ý định hành vi có ảnh hưởng trực tiếp đến Hành vi sử dụng hệ thống e-learning của nhân viên. Tương tự, trong các khám phá của Nguyen và cộng sự (2014) minh họa rằng Ý định hành vi có ảnh hưởng tích cực đến Hành vi sử dụng hệ thống e-learning dựa trên điện toán đám mây. Pham và cộng sự (2020) chỉ ra rằng Ý định hành vi có ảnh hưởng mạnh đến Hành vi sử dụng của giáo viên. Dựa vào các báo cáo trên, tác giả đưa ra giải thuyết:

H7: Ý định hành vi (Behavioral intention) sẽ có ảnh hưởng trục tiếp đến Hành vi sủ dụng (Use behavior) phần mềm Portal Office của viên chưcc

\subsubsection{Mô hình đề xuất}

Tập trung vào việc phân tích ảnh hưởng của các nhân tố và các mối quan hệ của chúng đến Ý định hành vi và Hành vi sử dụng phần mềm Portal Office của viên chức trường Đại học Quốc tế (ĐHQT) từ các mô hình nghiên cứu khác như: UTAUT, UTAUT2, TAM và kết quả các nghiên cứu thực nghiệm, tác giả xây dựng mô hình đề xuất như sau: 


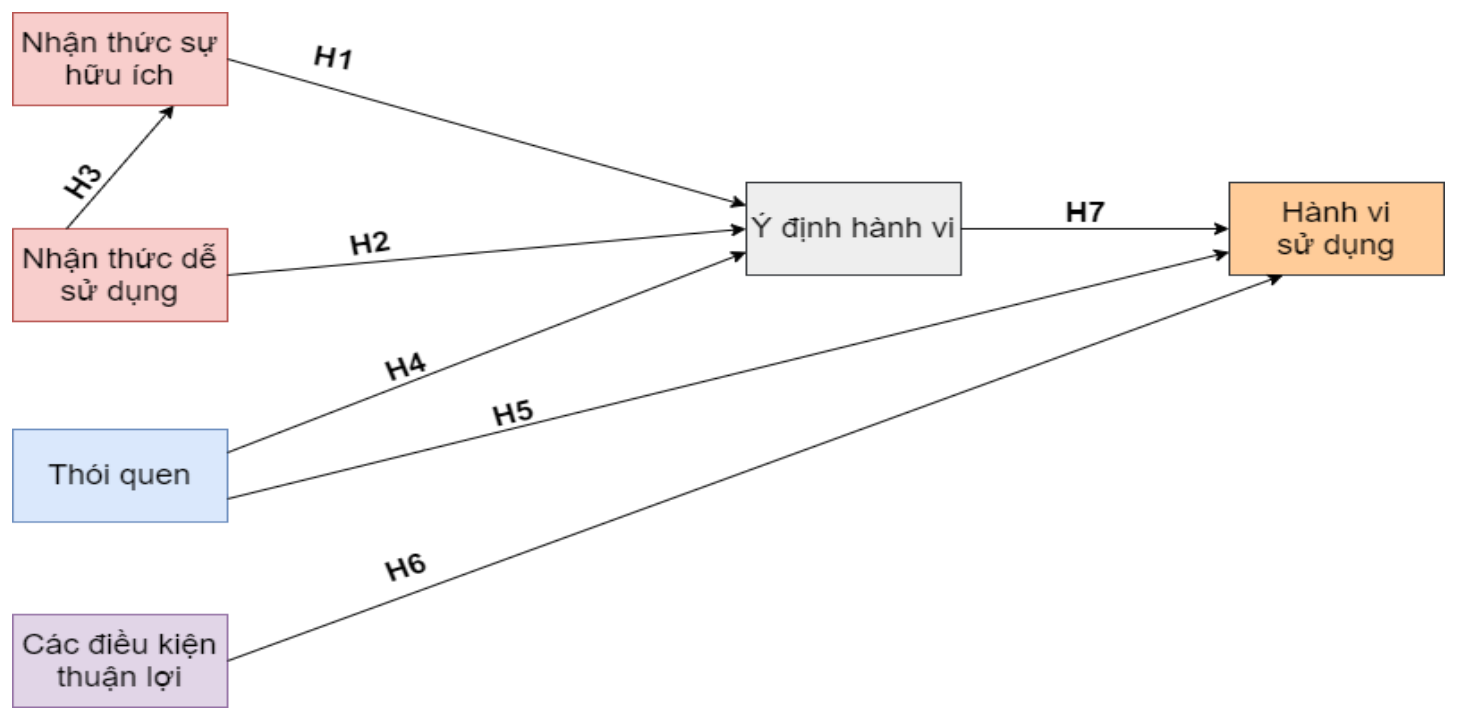

Hình 1. Mô hình nghiên cứu đề xuất

\section{Phương pháp nghiên cứu}

Nghiên cứu này sử dụng phương pháp nghiên cứu định lượng. Quy trình thực hiện thông qua các bước khảo cứu các khái niệm, các nghiên cứu liên quan để xây dựng mô hình nghiên cứu. Sau đó, tham khảo và kế thừa các bộ công cụ từ những nghiên cứu trước để đề xuất thang đo sơ bộ. Tác giả phỏng vấn trực tiếp và thu thập các ý kiến đóng góp, chỉnh sửa từ 20 đáp viên để điều chỉnh bảng câu hỏi. Thang đo chi tiết được mô tả ở Bảng 1 bao gồm Nhận thức sự hữu ích (06 quan sát), Nhận thức dễ sử dụng (05 quan sát), Các điều kiện thuận lợi (04 quan sát), Thói quen (04 quan sát), Ý định hành vi (05 quan sát) và Hành vi sử dụng (05 quan sát). Phương pháp lấy mẫu được đề xuất là lấy mẫu thuận tiện để chọn các đối tượng khảo sát. Cỡ mẫu bị ảnh hưởng bởi một số yếu tố, bao gồm mục đích của nghiên cứu, nhân khẩu, rủi ro lựa chọn một mẫu lỗi và sai số lấy mẫu cho phép (Israel, 1992). Đối với nghiên cứu này, với những giới hạn về chi phí, nhân khẩu và thời gian, kích thước mẫu sẽ được lấy ở mức tối ưu cần thiết. Theo Hair, Black, Babin, và Anderson (2009), kỹ thuật phân tích nhân tố khám phá EFA đề xuất kích thước mẫu tối thiểu gấp 05 lần tổng số biến quan sát. Kline (2014) khuyến nghị kích thước mẫu ít nhất là 100 , tuy nhiên, việc xem xét tỷ lệ số lượng người tham gia so với số lượng các biến là một cách tốt hơn để xác định cỡ mẫu tối thiểu. Gorsuch (1974) khuyến nghị một tỷ lệ tối thiểu có thể chấp nhận được là không ít hơn 100 cá thể cho bất kỳ phân tích nào. Theo đó, nghiên cứu chọn kích thước mẫu tối thiểu là $\mathrm{N}=100$, tác giả đã truy cập vào dữ liệu hệ thống phần mềm Portal Office, sau đó chọn lọc ra đối tượng được tiến hành khảo sát là các viên chức có lịch sử đã từng đăng nhập vào hệ thống, 169 bảng câu hỏi trong đó có 104 bảng theo hình thức in giấy và 65 bảng câu hỏi điện tử được gửi đến trực tiếp cho các đối tượng đã được chọn lọc thông qua việc gặp, phân phát trực tiếp hoặc gửi email có nội dung bảng câu hỏi từ Tháng 03 đến Tháng 04 năm 2021 . Kết quả thu về 108 phản hồi. Số phản hồi hợp lệ là 100 . Dữ liệu sau khi được thu thập và làm sạch được đưa vào phân tích. SEM (Structural Equation Model) là một trong những kỹ thuật phân tích thống kê tiên tiến nhất xuất hiện trong những thập kỷ gần đây (Hair, Hult, Ringle, \& Sarstedt, 2016). Đây là một loại kỹ thuật đa biến kết hợp các khía cạnh của phân tích nhân tố và hồi quy, cho phép các nhà nghiên cứu đồng thời kiểm tra mối quan hệ giữa các cấu trúc tiềm ẩn như Nhận thức, Thái độ hoặc Ý định của Người tiêu dùng và ảnh hưởng của chúng đến các thước đo Hiệu suất Tổ chức. Phổ biến nhất có hai cách tiếp cận để ước tính các mối quan hệ trong mô hình phương trình cấu trúc là CB-SEM sử dụng khi dữ liệu phân phối chuẩn, có cở mẫu lớn và PLS-SEM sử dụng với cở mẫu nhỏ và không giả định phân phối chuẩn (Hair \& ctg., 2016; Hair, Risher, Sarstedt, \& Ringle, 2019). Mỗi kỹ thuật phân tích phù hợp với một mục tiêu nghiên cứu khác nhau. Trong thời gian 
gần đây, PLS-SEM hiện được ứng dụng rộng rãi trong nhiều ngành khoa học xã hội, bao gồm quản lý tổ chức (Sosik, Kahai, \& Piovoso, 2009), quản lý nguồn nhân lực (Ringle, Sarstedt, Mitchell, \& Gudergan, 2020), hệ thống thông tin quản lý (Hair, Hollingsworth, Randolph, \& Chong, 2017; Ringle, Sarstedt, \& Straub, 2012), quản lý hoạt động (Peng \& Lai, 2012). Phương pháp PLS-SEM sẽ được sử dụng cho phù hợp xử lý cỡ mẫu nhỏ. Để đánh giá kết quả PLS-SEM bao gồm việc kiểm tra các mô hình đo lường. Nếu các mô hình đo lường đáp ứng yêu cầu, các nhà nghiên cứu cần đánh giá mô hình cấu trúc (Hair \& ctg., 2017).

\section{Bảng 1}

Thang đo đề xuất

\begin{tabular}{|c|c|c|}
\hline $\begin{array}{l}\text { Mã } \\
\text { hóa }\end{array}$ & Nội dung câu hỏi & Nguồn \\
\hline \multicolumn{3}{|c|}{ Nhận thức sự hữu ích } \\
\hline HI1 & $\begin{array}{l}\text { Sử dụng Portal Office trong công việc cho phép tôi } \\
\text { hoàn thành nhiệm vụ nhanh hơn }\end{array}$ & \multirow{2}{*}{ Davis và cộng sự (1989) } \\
\hline HI3 & $\begin{array}{l}\text { Sử dụng Portal Office làm tăng năng suất làm việc của } \\
\text { tôi }\end{array}$ & \\
\hline $\mathrm{HI} 2$ & $\begin{array}{l}\text { Sử dụng Portal Office cải thiện hiệu suất công việc của } \\
\text { tôi (tránh lãng phí công sức, thời gian) }\end{array}$ & \multirow{2}{*}{$\begin{array}{l}\text { Davis và cộng sự (1989); } \\
\text { Tapanainen, Dao, Nguyen, và } \\
\text { Nguyen (2019) }\end{array}$} \\
\hline HI6 & Tôi thấy Portal Office hữu ích trong công việc của tôi & \\
\hline HI4 & Sử dụng Portal Office giúp tôi tăng hiệu quả công việc & $\begin{array}{l}\text { Chan (2004); Davis và cộng sự } \\
\text { (1989); Tapanainen và cộng sự } \\
\text { (2019) }\end{array}$ \\
\hline HI5 & $\begin{array}{l}\text { Sử dụng Portal Office giúp tôi thực hiện công việc dễ } \\
\text { hơn }\end{array}$ & $\begin{array}{l}\text { Chan (2004); Davis và cộng sự } \\
(1989)\end{array}$ \\
\hline \multicolumn{3}{|c|}{ Nhận thức dễ sử dụng } \\
\hline DD1 & Học cách vận hành Portal Office khá dễ dàng đối với tôi & \multirow{2}{*}{$\begin{array}{l}\text { Chan (2004); Davis và cộng sự } \\
\text { (1989) }\end{array}$} \\
\hline DD3 & $\begin{array}{l}\text { Tôi thấy Portal Office thật rõ ràng và dễ hiểu để tương } \\
\text { tác }\end{array}$ & \\
\hline DD2 & $\begin{array}{l}\text { Tôi thấy thật dễ quản lý, tìm kiếm, nhận thông báo văn } \\
\text { bản thông qua Portal Office }\end{array}$ & Davis và cộng sự (1989) \\
\hline DD4 & Tôi thấy dễ thành thạo Portal Office & \multirow{2}{*}{$\begin{array}{l}\text { Chan (2004); Davis và cộng sự } \\
\text { (1989); Tapanainen và cộng sự } \\
(2019)\end{array}$} \\
\hline DD5 & Tôi thấy Portal Office dễ sử dụng & \\
\hline \multicolumn{3}{|c|}{ Các điều kiện thuận lọii } \\
\hline TL1 & $\begin{array}{l}\text { Trường ĐHQT có cơ sở hạ tầng kỹ thuật cần thiết để hỗ } \\
\text { trợ tôi sử dụng Portal Office }\end{array}$ & \multirow{2}{*}{$\begin{array}{l}\text { Nguyen và cộng sự (2014); } \\
\text { Pham và cộng sự (2020) } \\
\text { Venkatesh và cộng sự (2003); } \\
\text { Venkatesh và cộng sự (2012); } \\
\text { Wang (2016); Wong và cộng } \\
\text { sự (2013) }\end{array}$} \\
\hline TL2 & $\begin{array}{l}\text { Tôi có kiến thức công nghệ thông tin cần thiết để sử } \\
\text { dụng hệ thống Portal Office }\end{array}$ & \\
\hline
\end{tabular}




\begin{tabular}{|c|c|c|}
\hline $\begin{array}{l}\text { Mã } \\
\text { hóa }\end{array}$ & Nội dung câu hỏi & Nguồn \\
\hline TL3 & $\begin{array}{l}\text { Portal Office không tương thích với các hệ thống khác } \\
\text { mà tôi sử dụng }\end{array}$ & $\begin{array}{l}\text { Venkatesh và cộng sự (2003); } \\
\text { Venkatesh và cộng sự (2012); } \\
\text { Wang (2016) }\end{array}$ \\
\hline TL4 & $\begin{array}{l}\text { Có người sẵn sàng hỗ trợ khi tôi gặp khó khăn trong lúc } \\
\text { sử dụng Portal Office }\end{array}$ & $\begin{array}{l}\text { Thompson, Higgins, và } \\
\text { Howell (1991); Venkatesh và } \\
\text { cộng sự (2003); Venkatesh và } \\
\text { cộng sự (2012); Wong và cộng } \\
\text { sự (2013) }\end{array}$ \\
\hline \multicolumn{3}{|c|}{ Thói quen } \\
\hline TQ1 & $\begin{array}{l}\text { Việc sử dụng Portal Office đã trở thành một thói quen } \\
\text { đối với tôi }\end{array}$ & \multirow{3}{*}{$\begin{array}{l}\text { Nguyen và cộng sự (2014); } \\
\text { Pham và cộng sự (2020); } \\
\text { Venkatesh và cộng sự (2003) }\end{array}$} \\
\hline TQ2 & Tôi sử dụng Portal Office thường xuyên & \\
\hline TQ3 & $\begin{array}{l}\text { Nếu không có yếu tố bắt buộc, tôi vẫn thấy nhất thiết } \\
\text { cần phải sử dụng Portal Office }\end{array}$ & \\
\hline TQ4 & $\begin{array}{l}\text { Sử dụng Portal Office đã trở thành điều tự nhiên đối với } \\
\text { tôi }\end{array}$ & $\begin{array}{l}\text { Pham và cộng sự (2020); } \\
\text { Venkatesh và cộng sự (2012) }\end{array}$ \\
\hline \multicolumn{3}{|c|}{ Ý định hành vi } \\
\hline YD1 & $\begin{array}{l}\text { Tôi sẽ sử dụng Portal Office bất cứ lúc nào tôi có nhu } \\
\text { cầu }\end{array}$ & $\begin{array}{l}\text { Davis (1993); Venkatesh và } \\
\text { Davis (2000); Wang (2016); } \\
\text { Wong và cộng sự (2013) }\end{array}$ \\
\hline YD2 & $\begin{array}{l}\text { Tôi nghĩ nên khuyến khích mọi người sử dụng Portal } \\
\text { Office }\end{array}$ & \multirow{2}{*}{$\begin{array}{l}\text { Davis (1993); Tapanainen và } \\
\text { cộng sự (2019); Venkatesh và } \\
\text { Davis (2000) }\end{array}$} \\
\hline YD5 & Tôi sẽ giới thiệu Portal Office cho người khác & \\
\hline YD3 & $\begin{array}{l}\text { Tôi sẽ thường xuyên sử dụng Portal Office trong tương } \\
\text { lai }\end{array}$ & $\begin{array}{l}\text { Ajzen (1991); Davis và cộng } \\
\text { sự (1989); Moore và Benbasat } \\
\text { (1991); Wang (2016); Wong } \\
\text { và cộng sự (2013) }\end{array}$ \\
\hline YD4 & $\begin{array}{l}\text { Tôi nghĩ hầu hết mọi nhiệm vụ của tôi đều thực hiện } \\
\text { được thông qua Portal Office }\end{array}$ & $\begin{array}{l}\text { Ajzen (1991); Moore và } \\
\begin{array}{l}\text { Benbasat (1991); Pham và } \\
\text { cộng sự }(2020)\end{array} \\
\end{array}$ \\
\hline \multicolumn{3}{|c|}{ Hành vi sử dụng } \\
\hline HV1 & Tôi có thể sử dụng Portal Office cho mọi nhiệm vụ & \multirow{5}{*}{$\begin{array}{l}\text { Davis (1993); Tapanainen và } \\
\text { cộng sự (2019); Venkatesh và } \\
\text { Davis (2000) }\end{array}$} \\
\hline HV2 & Tôi sử dụng Portal Office cho nhiều hơn một nhiệm vụ & \\
\hline HV3 & $\begin{array}{l}\text { Tôi sẽ tiếp tục sử dụng Portal Office cho công việc } \\
\text { trong thời gian tới }\end{array}$ & \\
\hline HV4 & Nhìn chung Portal Office làm tôi thấy hài lòng & \\
\hline HV5 & $\begin{array}{l}\text { Tôi thấy thích khi sử dụng Portal Office cho công việc } \\
\text { của mình }\end{array}$ & \\
\hline
\end{tabular}

Nguồn: Tác giả tổng hợp (2021) 


\section{Kết quả phân tích và thảo luận}

\subsection{Mô tả đăc điểm mẫu}

Nghiên cứu chính thức với số bảng câu hỏi được gửi đi là 169 , tổng số lượng đáp viên phản hồi là 108 (tỷ lệ phản hồi 64\%). Sau khi tác giả tiến hành lọc và xử lý dữ liệu, số mẫu hợp lệ cuối cùng chính thức là $\mathrm{N}=100$. Về đặc điểm giới tính, dựa theo kết quả khảo sát, giới tính Nữ chiếm nhiều hơn với tỷ lệ $53 \%$. Có thể thấy sự chênh lệch về giới tính không đáng kể. Về đặc điểm độ tuổi, kết quả cho thấy mức tuổi tác từ 30 - 40 tuổi chiếm tỷ lệ đa số $67 \%$; từ 23 - 29 tuổi chiếm $25 \%$, và trên 40 tuổi chiếm $8 \%$. Như vậy, có thể thấy các mức độ tuổi tương đối có sự chênh lệch lớn. Đối với đặc điểm trình độ học vấn, trình độ cử nhân/kỹ sư chiếm $51 \%$; trình độ thạc sĩ chiếm $46 \%$; trình độ tiến sĩ chiếm $3 \%$. Xem xét đặc điểm về bằng cấp/chứng chỉ tin học, kết quả cho thấy đáp viên có bằng cấp/chứng chỉ về tin học chiếm tỷ lệ $69 \%$; đáp viên chưa có bằng cấp/chứng chỉ về công nghệ thông tin chiếm tỷ lệ $31 \%$. Đối với đặc điểm về tham gia lớp tập huấn Portal Office, đáp viên có cơ hội tham gia huấn luyện về sử dụng Portal Office chiếm tỷ lệ $74 \%$; đáp viên chưa từng tham gia chiếm tỷ lệ $26 \%$.

\subsection{Kiểm định độ tin cậy thang đo}

Các hệ số tải về độ tin cậy phải đáp ứng được giá trị vượt qua mức chỉ số 0.7 (Hair \& ctg., 2016). Các kết quả chỉ ra nhân tố $\mathrm{HI}$ (Nhận thức sự hữu ích) có 05 biến quan sát với hệ số nhân tải ngoài trong khoảng 0.904 - 0.928; nhân tố DD (Nhận thức dễ sử dụng) có 05 biến quan sát với hệ số nhân tải ngoài trong khoảng 0.739 - 0.913; nhân tố TQ (Thói quen) có 04 biến quan sát với hệ số nhân tải ngoài trong khoảng 0.827 - 0.913; nhân tố TL (Các điều kiện thuận lợi) với 02 biến quan sát với hệ số nhân tải ngoài trong khoảng $0.719-0.905$; nhân tố YD (Ý định hành vi) có 05 biến quan sát với hệ số nhân tải ngoài trong khoảng $0.722-0.909$; nhân tố HV (Hành vi sử dụng) có 04 biến quan sát với hệ số nhân tải ngoài trong khoảng $0.868-0.924$. Tiếp theo đó, theo Hair và cộng sự (2019) độ tin cậy tổng hợp CR thỏa đáng trong khoảng 0.7 đến 0.9 . Phương sai trích AVE lớn hơn giá trị ngưỡng 0.5. Độ tin cậy Cronbach's Alpha $>=0.7$. Kết quả phân tích cho thấy tất cả các nhân tố thể hiện Độ tin cậy tổng hợp $(\mathrm{CR})$ đều đạt yêu cầu với hệ số lớn hơn 0.7 và cụ thể là nằm trong khoảng giá trị từ $0.799-0.963$. Hệ số Phương sai trích $\mathrm{AVE}$ của các nhân tố đều nằm trong khoảng 0.668 - 0.838 và lớn hơn 0.5 giải thích được tối thiểu $>$ $50 \%$ biến thiên của dữ liệu quan sát nên thang đo đạt giá trị hội tụ. Độ tin cậy Cronbach's Alpha đều trong khoảng từ 0.890 - 0.952. Riêng nhân tố TL (Các điều kiện thuận lợi) có $\mathrm{CA}=0.623$ (> 0.5) sự thay đổi giá trị theo Nunnally và Bernstein (1994). Để đảm bảo không có mối quan hệ tương quan nào giữa các yếu tố được sử dụng để đo lường các nhân tố, Độ giá trị phân biệt được kiểm định bởi Giá trị phân biệt (Discriminant validity). Theo Hair và cộng sự (2019), Giá trị phân biệt được xác định bằng cách bình phương $\mathrm{AVE}$, độ gi á trị phân biệt được bảo đảm khi hệ số tải của các nhân tố này là lớn nhất so với các nhân tố khác.

\section{Bảng 2}

Kết quả kiểm định Giá trị phân biệt

\begin{tabular}{|l|c|c|c|c|c|c|}
\hline \multicolumn{1}{|c|}{ Nhân tố } & DD & HI & HV & TL & TQ & YD \\
\hline DD (Nhận thức dễ sử dụng) & $\mathbf{0 . 8 5 4}$ & & & & & \\
\hline HI (Nhận thức sự hữu ích) & 0.475 & $\mathbf{0 . 9 1 6}$ & & & & \\
\hline HV (Hành vi sử dụng) & 0.600 & 0.759 & $\mathbf{0 . 9 0 1}$ & & & \\
\hline TL (Các điều kiện thuận lợi) & 0.473 & 0.410 & 0.536 & $\mathbf{0 . 8 1 7}$ & & \\
\hline TQ (Thói quen) & 0.647 & 0.719 & 0.811 & 0.483 & $\mathbf{0 . 8 8 5}$ & \\
\hline YD (Ý định hành vi) & 0.636 & 0.698 & 0.866 & 0.496 & 0.831 & $\mathbf{0 . 8 3 5}$ \\
\hline
\end{tabular}

Nguồn: Kết quả phân tích dữ liệu 


\subsection{Kiểm định mô hình cấu trúc}

Không có một tiêu chí nào là duy nhất phù hợp để đánh giá các ước tính trong PLS-SEM. Việc đánh giá kết quả đo lường và mô hình cấu trúc trong PLS-SEM được xây dựng dựa trên một tập hợp các tiêu chí đánh giá phi tham số và sử dụng các kỹ thuật chẳng hạn như bootstrapping (Hair \& ctg., 2019). Trong cuộc khảo sát nghiên cứu này, mỗi mẫu bootstrapping sẽ chứa 100 quan sát tương ứng với tổng số quan sát của mẫu ban đầu. Nhằm bảo đảm được yêu cầu kiểm định của mô hình cấu trúc tuyến tính, tác giả triển khai kỹ thuật bootstrapping 1,000 lần (Hair \& ctg., 2016).

\subsection{Kiểm định vi phạm đa cộng tuyến (Collinearity Statistics)}

Để đánh giá các vấn đề liên kết trong mô hình cấu trúc cần dựa vào kết quả quan trọng duy nhất là giá trị dung sai (VIF). VIF của mỗi chỉ báo phải cao hơn 0.2 và thấp hơn 5 . Nếu nằm ngoài giới hạn này, cần xem xét loại bỏ các chỉ số, hợp nhất các chỉ số thành một chỉ mục duy nhất hoặc tạo các cấu trúc bậc cao hơn để xử lý các vấn đề về đa cộng tuyến (Hair \& ctg., 2019). Bảng 3 cho thấy hệ số VIF chỉ ra rằng không có vi phạm về giả định về đa cộng tuyến đối với sự liên kết giữa các nhân tố dự đoán vì tất cả các hệ số đều nằm trong khoảng chấp nhận (VIF = 1.144 - $4.613<5)$, do đó có thể tiếp tục việc phân tích.

\section{Bảng 3}

Kết quả kiểm định đa cộng tuyến

\begin{tabular}{|l|l|l|}
\hline \multicolumn{1}{|c|}{ Nhân tố } & \multicolumn{1}{c|}{ Hệ số phóng đại phương sai (VIF) } & \multicolumn{1}{c|}{ Đánh giá } \\
\hline DD (Nhận thức dễ sử dụng) & $1.858-1.935$ & $<5:$ Không vi phạm \\
\hline HI (Nhận thức sự hữu ích) & $3.662-4.613$ & $<5:$ Không vi phạm \\
\hline HV (Hành vi sử dụng) & $2.548-3.834$ & $<5:$ Không vi phạm \\
\hline TL (Các điều kiện thuận lợi) & $1.144-1.144$ & $<5:$ Không vi phạm \\
\hline TQ (Thói quen) & $2.011-3.942$ & $<5:$ Không vi phạm \\
\hline YD (Ý định hành vi) & $1.670-4.104$ & $<5:$ Không vi phạm \\
\hline
\end{tabular}

Nguồn: Kết quả phân tích dữ liệu

\subsection{Kiểm định sụ phù hợp mô hình (model fit)}

Có một số các tiêu chí chính nhằm thực hiện việc đánh giá mô hình cấu trúc trong PLSSEM bao gồm: các hệ số đường dẫn (path coefficients), giá trị $\mathrm{R}^{2}$, hệ số tác động $\mathrm{f}^{2}$, mức độ phù hợp dự báo $Q^{2}$ (Hair \& ctg., 2019). Thước đo thường được sử dụng nhất để đánh giá mô hình cấu trúc là hệ số xác định (giá trị $\mathrm{R}^{2}$ ). Giá trị $\mathrm{R}^{2}$ càng cao sẽ cho thấy mức độ dự đoán càng chính xác. Kết quả phân tích cho thấy, giá trị $\mathrm{R}^{2}$ của mô hình $\mathrm{HI}$ là 0.225 ; giá trị $\mathrm{R}^{2}$ của mô hình $\mathrm{HV}$ là 0.787 và $Y D$ với $\mathrm{R}^{2}$ của mô hình là 0.728 . Ngoài ra, giá trị $\mathrm{Q}^{2}$ của Stone-Geisser (Geisser, 1974; Stone, 1974) là một chỉ báo về mức độ phù hợp dự đoán của mô hình. Trong mô hình cấu trúc, các giá trị $Q^{2}$ lớn hơn 0 cho một biến tiềm ẩn nội sinh phản ánh mức độ liên quan nhất định dự đoán của mô hình đường dẫn đối với cấu trúc cụ thể này (Hair \& ctg., 2019). Bảng 4 cho thấy $\mathrm{Q}^{2}$ của kết quả nghiên cứu này đối với tất cả các cấu trúc có giá trị không nhỏ hơn 0 , như vậy có nghĩa là tất cả biến phụ thuộc trong mô hình đề xuất đều có mức độ phù hợp cho dự đoán. 


\section{Bảng 4}

Kết quả của $\mathrm{R}^{2}$ và $\mathrm{Q}^{2}$

\begin{tabular}{|l|c|c|}
\hline & Giá trị R & Giá trị $\mathrm{Q}^{2}$ \\
\hline HI (Nhận thức sự hữu ích) & 0.225 & 0.187 \\
\hline HV (Hành vi sử dụng) & 0.787 & 0.628 \\
\hline YD (Ý định hành vi) & 0.728 & 0.489 \\
\hline
\end{tabular}

Nguồn: Kết quả phân tích dữ liệu

Ngoài việc đánh giá các giá trị $\mathrm{R}^{2}$ trong cấu trúc nội sinh, trong trường hợp một cấu trúc ngoại sinh thay đổi làm thay đổi $\mathrm{R}^{2}$, sự thay đổi bị bỏ qua này có thể được sử dụng để đánh giá xem cấu trúc bị bỏ qua có tác động đáng kể đến cấu trúc nội sinh hay không. Phép đo lường này được gọi là hệ số tác động $\mathrm{f}^{2}$. Các giá trị $\mathrm{f}^{2}$ lần lượt là $0.02 ; 0.15$ và 0.35 phản ánh các tác động "nhỏ", "trung bình" và "lớn" (Cohen, 2013). Kết quả Bảng 5 cho thấy:

\section{Bảng 5}

Kết quả giá trị $\mathrm{f}^{2}$ và mức độ ảnh hưởng

\begin{tabular}{|c|c|c|}
\hline Mối quan hệ giữa các biến & Giá trị f $^{2}$ & Mức độ ảnh hưởng \\
\hline $\mathbf{T L} \rightarrow \mathbf{H V}$ & 0.047 & Nhỏ \\
\hline $\mathbf{D D} \rightarrow \mathbf{Y D}$ & 0.059 & Nhỏ \\
\hline $\mathbf{H I} \rightarrow \mathbf{Y D}$ & 0.075 & Nhỏ \\
\hline $\mathbf{T Q} \rightarrow \mathbf{H V}$ & 0.104 & Trung bình \\
\hline $\mathbf{D D} \rightarrow \mathbf{H I}$ & 0.291 & Trung bình \\
\hline TQ $\rightarrow$ YD & 0.443 & Lớn \\
\hline $\mathbf{Y D} \rightarrow \mathbf{H V}$ & 0.477 & Lớn \\
\hline
\end{tabular}

Nguồn: Kết quả phân tích dữ liệu

\subsection{Kiểm định mô hình cấu trúc tuyến tính}

Trong nghiên cứu này, tác giả thực hiện bootstrapping phi tham số kiểm định mẫu 100 quan sát, thiết lập mức lặp 1,000 lần nhằm đáp ứng yêu cầu kiểm định mô hình cấu trúc tuyến tính. Kết quả kiểm định mối quan hệ giữa các biến có thể thấy được ở bảng 6 dưới đây:

\section{Bảng 6}

Kết quả tác động trực tiếp của các mối quan hệ

\begin{tabular}{|c|c|c|c|c|}
\hline Mối quan hệ giữa các biến & Hệ số tác động & Sai số (SE) & Giá trị t & Mức ý nghĩa \\
\hline TQ $\rightarrow$ YD & 0.576 & 0.095 & 6.056 & 0.000 \\
\hline HI $\rightarrow$ YD & 0.205 & 0.099 & 2.071 & 0.039 \\
\hline $\mathbf{D D} \rightarrow$ YD & 0.166 & 0.072 & 2.302 & 0.022 \\
\hline TQ $\rightarrow$ HV & 0.270 & 0.098 & 2.758 & 0.006 \\
\hline TL $\rightarrow$ HV & 0.116 & 0.054 & 2.150 & 0.032 \\
\hline $\mathbf{D D} \rightarrow \mathbf{H I}$ & 0.475 & 0.083 & 5.684 & 0.000 \\
\hline YD $\rightarrow$ HV & 0.584 & 0.103 & 5.671 & 0.000 \\
\hline
\end{tabular}

Nguồn: Kết quả phân tích dữ liệu 
Kết quả cho thấy mô hình Ý định hành vi sử dụng Portal Office có 03 mối quan hệ cùng chiều và có ý nghĩa thống kê ở độ tin cậy $95 \%$ là: (1) $\mathbf{T Q} \rightarrow \mathbf{Y D}$ với hệ số tác động $(\beta=0.576)$; (2) $\mathbf{H I} \rightarrow$ YD với hệ số tác động $(\beta=0.205)$; (3) $\mathbf{D D} \rightarrow \mathbf{Y D}$ với hệ số tác động $(\beta=0.166)$. So sánh mức độ 03 biến $\mathrm{TQ}, \mathrm{DD}, \mathrm{HI}$ tác động vào biến phụ thuộc Ý định hành vi sử dụng YD theo thứ tự giảm dần, ta thây: biến TQ (Thói quen) có tác động mạnh nhất $(\beta=0.576)$, tiếp theo là biến HI (Nhận thức sự hữu ích) $(\beta=0.205)$ và cuối cùng là biến $\mathrm{DD}$ (Nhận thức dễ sử dụng) ( $\beta=$ 0.166). Như vậy các giả thuyết được chấp nhận là $\mathrm{H} 1, \mathrm{H} 2, \mathrm{H} 4$ với độ tin cậy $95 \%$. Điều này cho thấy Thói quen, Nhận thức dễ sử dụng, Nhận thức sự hữu ích đều tạo nên Ý định hành vi sử dụng Portal Office.

Mô hình Hành vi sử dụng Portal Office có 02 mối quan hệ cùng chiều và có ý nghĩa thống kê ở độ tin cậy $95 \%$ là: (1) $\mathbf{T Q} \rightarrow \mathbf{H V}$ với hệ số tác động $(\beta=0.270)$; (2) $\mathbf{T L} \rightarrow \mathbf{H V}$ với hệ số tác động $(\beta=0.116)$. So sánh mức độ 02 biến $T Q$, TL tác động vào biến phụ thuộc Hành vi sử dụng HV theo chiều hướng giảm dần, ta thấy: biến TQ (Thói quen) có tác động mạnh nhất ( $\beta$ $=0.270)$, tiếp đến là biến $\mathrm{TL}$ (Các điều kiện thuận lợi) $(\beta=0.116)$. Như vậy các giả thuyết chấp nhận được ở độ tin cậy $95 \%$ bao gồm H5, H6. Theo đó, Thói quen và Các điều kiện thuận lợi có sự ảnh hưởng đến Hành vi sử dụng Portal Office.

Xét mô hình mối quan hệ giữa 02 biến $\mathrm{DD}, \mathrm{HI}$, ta có 01 mối quan hệ cùng chiều và có ý nghĩa thống kê ở độ tin cậy $99 \%$ là: $\mathbf{D D} \rightarrow \mathbf{H I}$ với hệ số tác động $(\beta=0.475)$. Như vậy, giả thuyết $\mathrm{H3}$ được chấp nhận với độ tin cậy $99 \%$. Có nghĩa là Nhận thức dễ sử dụng có tác động đáng kể đến Nhận thức sự hữu ích.

Trong nghiên cứu này, tác giả cũng nhìn nhận mối quan hệ giữa Ý định hành vi và Hành vi sử dụng, kết quả cho thấy đây là 02 mối quan hệ tương quan cùng chiều và có sự ảnh hưởng đáng kể, cụ thể như sau: YD $\rightarrow \mathbf{H V}$ với hệ số tác động $(\beta=0.584)$ có ý nghĩa thống kê ở độ tin cậy $99 \%$. Do đó, H7 được chấp nhận ở độ tin cậy $99 \%$. Như vậy, Ý định hành vi có ảnh hưởng trực tiếp đến Hành vi sử dụng.

Tác giả cũng xem xét tác động gián tiếp của các nhân tố đến Hành vi sử dụng (HV) thông qua Ý định hành vi (YD). Phân tích SmartPLS đã ra các kết quả cho thấy các mối qu an hệ gi án tiếp như sau:

\section{Bảng 7}

Kết quả tác động gián tiếp của các nhân tố

\begin{tabular}{|c|c|c|c|c|}
\hline Mối quan hệ giữa các biến & Hệ số Beta & Sai số $(\mathbf{S E})$ & Giá trị t & Mức ý nghĩa \\
\hline $\mathrm{TQ} \rightarrow \mathrm{YD} \rightarrow \mathrm{HV}$ & 0.337 & 0.078 & 4.337 & 0.000 \\
\hline $\mathrm{DD} \rightarrow \mathrm{YD} \rightarrow \mathrm{HV}$ & 0.097 & 0.043 & 2.233 & 0.026 \\
\hline $\mathrm{DD} \rightarrow \mathrm{HI} \rightarrow \mathrm{YD}$ & 0.097 & 0.046 & 2.132 & 0.033 \\
\hline $\mathrm{DD} \rightarrow \mathrm{HI} \rightarrow \mathrm{YD} \rightarrow \mathrm{HV}$ & 0.057 & 0.030 & 1.871 & 0.062 \\
\hline $\mathrm{HI} \rightarrow \mathrm{YD} \rightarrow \mathrm{HV}$ & 0.120 & 0.065 & 1.840 & 0.066 \\
\hline
\end{tabular}

Nguồn: Kết quả phân tích dữ liệu

Từ Bảng 7 ta có thể thấy rằng 02 nhân tố TQ (Thói quen) và $\mathrm{DD}$ (Nhận thức dễ sử dụng) có tác động gián tiếp đến HV (Hành vi sử dụng) thông qua YD (Ý định hành vi), trong đó TQ có tác động lớn nhất $(\beta=0.337)$ với độ tin cậy $99 \%$, sau đó là $\mathrm{DD}(\beta=0.097)$ với độ tin cậy là $95 \%$. Bên cạnh đó, nhân tố $\mathrm{DD}$ có tác động gián tiếp đến YD thông qua $\mathrm{HI}(\beta=0.097)$ với độ tin cậy $95 \%$, đồng thời cũng có tác động gián tiếp đến $\mathrm{HV}$ thông qua $\mathrm{HI}$ và $\mathrm{YD}(\beta=0.057)$ với độ tin cậy $90 \%$. Cuối cùng, HI có tác động gián tiếp đến HV thông qua YD $(\beta=0.120)$ với độ tin cậy $90 \%$. 


\subsection{Thảo luận kết quả nghiên cúu}

Kết quả nghiên cứu đã chỉ ra rằng có mối quan hệ trực tiếp tác động của yếu tố Nhận thức sự hữu ích, Nhận thức dễ sử dụng đến Ý định hành vi (H1 và $\mathrm{H} 2)$. Đối với tác động gián tiếp lên Ý định hành vi, kết quả cho thấy Nhận thức dễ sử dụng có tác động gián tiếp lên Y̛ định hành vi thông qua Nhận thức sự hữu ích. Đối với tác động gián tiếp đến Hành vi sử dụng, ta có thể thấy cả 02 nhân tố Nhận thức sự hữu ích, Nhận thức dễ sử dụng đều có tác động gián tiếp đến Hành vi sử dụng thông qua Ý định hành vi. Điều này bổ sung thêm chứng cứ cho kết quả nghiên cứu của các tác giả Adams và cộng sự (1992); Agarwal và Karahanna (2000), Davis và cộng sự (1989), Doll và cộng sự (1998), Segars và Grover (1993). Nghiên cứu cũng xác định được mối quan hệ trực tiếp giữa Nhận thức dễ sử dụng và Nhận thức sự hữu ích (H3), đồng thời nhận thấy tác động gián tiếp của Nhận thức dễ sử dụng và Nhận thức sự hữu ích lên Hành vi sử dụng thông qua Ý định hành vi, cũng như tác động gián tiếp của Nhận thức dễ sử dụng lên Hành vi thông qua Nhận thức sự hữu ích và Ý định hành vi. Kết quả này tương đồng với Agarwal và Karahanna (2000), Gefen và Straub (2000), Hsu và Lu (2004), Lederer và cộng sự (2000), Moon và Kim (2001), Venkatesh và Davis (2000). Kết quả cho thấy Thói quen có sự trực tiếp tác động đến Ý định hành vi (H4) và Hành vi sử dụng (H5). Bên cạnh đó, Thói quen cũng gián tiếp có tác động lớn lên Hành vi sử dụng thông qua Ý định hành vi. Điều này thể hiện sự tương đồng với kết quả nghiên cứu của các tác giả Arenas và cộng sự (2015), Kim và Malhotra (2005), Kim và cộng sự (2005), Limayem và cộng sự (2007), Pham và cộng sự (2020). Cuối cùng, ta nhận thấy 02 nhân tố Các điều kiện thuận lợi và Ý định hành vi đều có tác động trực tiếp đến Hành vi sử dụng (H6, H7). Điều này phù hợp với kết quả nghiên cứu của Alalwan và cộng sự (2015), Im và cộng sự (2011), Nguyen và cộng sự (2014), Pham và cộng sự (2020), Teo (2011), Venkatesh và cộng sự (2003), và Wang (2016).

\section{Kết luận và khuyến nghị}

Các phát hiện cho thấy sự tác động giữa các yếu tố trong mô hình đề xuất đến Ý định và hành vi và Hành vi khi sử dụng phần mềm Portal Office. Trong đó, biến Thói quen có ảnh hưởng nhiều nhất đến Hành vi sử dụng. Hơn nữa, nghiên cứu cho thấy mối liên hệ chặt chẽ giữa Ý định hành vi và Hành vi sử dụng. Các phát hiện cũng phù hợp với các nghiên cứu trước đây trong cùng lĩnh vực. Kết quả nghiên cứu đã cung cấp thêm bằng chứng cho thấy các yếu tố ảnh hưởng đến Ý định và Hành vi sử dụng phần mềm Portal Office của viên chức trường ĐHQT bao gồm: Thói quen, Nhận thức sự hữu ích, Nhận thức dễ sử dụng và Các điều kiện thuận lợi.

Yếu tố Thói quen (TQ) có tác động mạnh nhất ( $\beta=0.576$ ) đến Ý định hành vi sử dụng, tiếp đó là đến Hành vi sử dụng $(\beta=0.270)$, điều này có ý nghĩa là khi viên chức của trường ĐHQT có thói quen sử dụng phần mềm Portal Office tăng thêm 01 lần thì Ý định sử dụng phần mềm của họ tăng thêm 0.576 lần, Hành vi sử dụng phần mềm của họ tăng thêm 0.270 lần (giả định rằng các biến khác không đổi). Cuối cùng, Thói quen có tác động gián tiếp đến Hành vi sử dụng thông qua Ý định hành vi sử dụng $(\beta=0.337)$. Độ lệch chuẩn của các biến quan sát Thói quen dao động ở mức cao từ $0.89-1.08$, giá trị trung bình không chênh lệch đáng kể từ 2.92 3.04. Do đó, có thể thấy sự biến thiên lớn ở các biến quan sát, thói quen sử dụng phần mềm Portal Office của viên chức tại trường ĐHQT là rất khác nhau. Vì vậy, tác giả có đề xuất với lãnh đạo các bộ phận của trường $\mathrm{OHQT}$ để tâm đến những vấn đề như cần tạo thói quen cho viên chức xử lý công việc thông qua việc sử dụng phần mềm Portal Office, bắt đầu bằng việc thay đổi theo hướng dần loại bỏ cách thức trao đổi và xử lý công việc theo lối truyền thống (ví dụ: cấp quản lý giao việc cho chuyên viên thông qua khẩu lệnh, văn bản giấy hoặc email). Nhà trường cũng cần truyền thông về phần mềm để củng cố thói quen sử dụng Portal Office của viên chức bằng cách khuyến khích, xét thi đua, không ngừng phổ biến về những lợi ích mà viên chức có được thông qua việc sử dụng phần mềm. 
Kết quả kiểm định cho thấy yếu tố Nhận thức sự hữu ích $(\beta=0.205)$ và Nhận thức dễ sử dụng $(\beta=0.166)$ đều có tác động trực tiếp đến Ý định hành vi sử dụng. Trong đó Nhận thức dễ sử dụng có ảnh hưởng đáng kể đến Nhận thức sự hữu ích $(\beta=0.475)$. Đối với mối quan hệ gián tiếp, ta có thể thấy yểu tố Nhận thức dễ dùng có sự gián tiếp tác động gián tiếp đến Hành vi sử dụng thông qua Ý định hành vi $(\beta=0.097)$ và thông qua Nhận thức sự hữu ích $\rightarrow Y$ Yịnh hành vi $\rightarrow$ Hành vi sử dụng $(\beta=0.057)$. Nhận thức sự hữu ích cũng tác động gián tiếp đến Hành vi sử dụng thông qua Ý định hành vi $(\beta=0.120)$. Kết quả này nhất quán với một số nghiên cứu trước đây và phù hợp với lý thuyết của mô hình TAM. Tổng trung bình các đánh giá của yếu tố Nhận thức sự hữu ích là mean $=3.452$ (độ lệch chuẩn từ 0.92 - 1.05); Tổng trung bình các đánh giá của yếu tố Nhận thức dễ sử dụng là mean $=3.502$ (độ lệch chuẩn từ $0.95-1.03$ ). Theo kết quả này, Ban lãnh đạo cần cải thiện một số điểm cụ thể như thảo luận với nhà cung cấp, nghiên cứu và tìm hiểu dựa trên cảm quan và nhu cầu sử dụng của người dùng, thiết kế Portal Office đảm bảo việc tận dụng khả năng nhận thức của nhân viên, nói cách khác là giao diện được đưa lên với các tính năng ở các vị trí trực quan, dễ thấy, dễ tương tác. Đối với một hệ thống nhiều tác vụ, điều này không nhất thiết phải làm cho nó trở nên dễ hiểu mà có nghĩa là thiết kế nó trở nên dễ dàng nhận biết và khám phá một cách nhanh chóng. Cần cố gắng đạt được việc đơn giản hóa và càng giúp người dùng tập trung vào các tác vụ chính của hệ thống càng tốt. Nhà trường cũng nên hiểu rõ sự cần thiết của Portal Office với các yêu cầu giải quyết công việc của nhân viên trong tương lai. Tóm lại, để tăng ý định hành vi sử dụng hệ thống Portal Office, cần phải phát triển hệ thống Portal Office trở nên khả dụng (có khả năng dễ sử dụng và tính ứng dụng cao). Khi ý định sử dụng hệ thống cao hơn, hành vi sử dụng sẽ theo đó tăng lên.

Có thể thấy yếu tố Các điều kiện thuận lợi $(\mathrm{TL})$ trong phạm vi nghiên cứu này (Mức ý nghĩa $95 \%)$ có sự tác động trực tiếp thấp nhất đến Hành vi sử dụng $(\beta=0.116)$ với mức ý nghĩa $95 \%$. Tuy nhiên đây cũng là một trong những thang đo quan trọng ảnh hưởng đến hành vi. Kết quả cho thấy mức độ đánh giá của viên chức về yếu tố Điều kiện thuận lợi tác động đến Hành vi sử dụng đều ở mức tương đối cao (mean từ 3.47 đến 3.97 với tổng trung bình là 3.84 ; độ lệch chuẩn từ $0.82-0.88$ ). Sự chênh lệch cũng không cao giữa các biến quan sát. Theo đó, tác giả đề xuất với Ban Giám Hiệu về hàm ý quản trị như trường $\mathrm{OHQT}$ cần phát triển cơ sở hạ tầng kỹ thuật nhằm ứng dụng Portal Office vào công việc một cách thuận lợi hơn (ví dụ: phát triển mạng lưới Internet tốt hơn, tối ưu hóa tốc độ đường truyền, nâng cấp hệ thống máy tính làm việc của nhân viên, tăng cường bảo mật, tăng cường các khóa đào tạo sử dụng hệ thống, ...).

Kết quả kiểm định cho thấy tác động của Ý định hành vi sử dụng phần mềm (YD) có tác động đáng kể $(\beta=0.584)$ đến Hành vi sử dụng. Ý định hành vi là một biến trung gian có tác động đáng kể đến dự đoán hành vi của người dùng. Theo đó, nghiên cứu này đề xuất: Để tăng khả năng ứng dụng Portal Office vào trong công việc của viên chức, nhà trường cần tăng Ý định hành vi người sử dụng thông qua việc nâng cao nhận thức rằng việc dùng phần mềm hỗ trợ xử lý công việc là hữu ích và dễ sử dụng, thói quen và các điều kiện thuận lợi. Quyết định sử dụng phần mềm với thói quen lặp lại theo thời gian có thể được thúc đẩy khi các nguồn lực và tài nguyên công nghệ sẵn có thúc đẩy việc sử dụng liên tục, chẳng hạn như các công cụ truyền thông, đào tạo, hướng dẫn sử dụng và các dịch vụ hỗ trợ kỹ thuật.

Nghiên cứu này bổ sung thêm các bằng chứng cho thấy UTAUT là mô hình phù hợp để giải thích ý định của viên chức trường $\mathrm{OHQT}$ về việc có nên ứng dụng Portal Office vào trong công việc hay không. Nghiên cứu cũng xem xét mô hình này trong bối cảnh cơ sở giáo dục và khu vực công tại Việt Nam. Kết quả khảo sát này sẽ hỗ trợ cho ban lãnh đạo có cái nhìn tổng quan về thực trạng việc ứng dụng công nghệ thông tin, giải quyết hồ sơ trên nền điện tử tại đơn vị của mình. Bên cạnh đó, thấy được những yếu tố nào đang ảnh hưởng đến ý thức, hành vi sử dụng phần mềm Portal Office để tìm ra các giải pháp đúng đắn và kịp thời để cải tiến hệ thống, 
cơ sở vật chất và kỹ thuật, tạo các điều kiện thuận lợi và thói quen nhằm thúc đẩy hành vi ứng dụng công nghệ vào nâng cao năng suất làm việc.

\section{Hạn chế của nghiên cứu và hướng nghiên cứu tiếp theo}

Thứ nhất, nghiên cứu này chỉ giới hạn đối tượng là viên chức trong bối cảnh cơ sở giáo dục trường ĐHQT đã từng có lịch sử đăng nhập phần mềm Portal Office, dẫn đến hạn chế lớn nhất là kích cỡ mẫu không lớn. Do đó, kết quả có thể không phản ánh nhận thức, hành vi của đại bộ phận viên chức ở các đơn vị khu vực công nói chung và các cơ sở giáo dục khác nói riêng. Tác giả đề xuất các nghiên cứu về sau sẽ kiểm tra mô hình được sử dụng cho viên chức trong các bối cảnh khác (các tỉnh/thành khác, các phần mềm khác) với tập mấu lớn hơn để xác minh tính đúng đắn của nó. Thứ hai, nghiên cứu này không phân tích tác động điều hòa của giới tính và tuổi tác, trải nghiệm và sự tự nguyện. Những ảnh hưởng này có thể hạn chế tính tổng quát của kết quả. Điều này đặt ra một vấn đề nghiên cứu tiềm năng cho tương lai là các nhà nghiên cứu có thể tiến hành các nghiên cứu khám phá và tìm hiểu vai trò điều tiết của chúng đối với ý định của viên chức về việc sử dụng phần mềm quản lý văn bản hành chính. Cuối cùng, các nghiên cứu trong tương lai có thể tích hợp thêm các yếu tố khác đối với mô hình UTAUT mà chưa đề cập đến trong nghiên cứu hoặc các mô hình khác để làm phong phú thêm khung khái niệm được sử dụng trong nghiên cứu này.

\section{LÒ̀I CÁM ONN}

Nghiên cứu này được trường Đại học Quốc tế, ĐHQG-HCM tài trợ trong đề tài có mã số SV2019-CPA-05.

\section{Tài liệu tham khảo}

Adams, D. A., Nelson, R. R., \& Todd, P. A. (1992). Perceived usefulness, ease of use, and usage of information technology: A replication. MIS Quarterly, 16(2), 227-247.

Agarwal, R., \& Karahanna, E. (2000). Time flies when you're having fun: Cognitive absorption and beliefs about information technology usage. MIS Quarterly, 24(4), 665-694.

Ajzen, I. (1991). The theory of planned behavior. Organizational Behavior \& Human Decision Processes, 50(2), 179-211.

Alalwan, A. A., Dwivedi, Y. K., Rana, N. P., Lal, B., \& Williams, M. D. (2015). Consumer adoption of Internet banking in Jordan: Examining the role of hedonic motivation, habit, self-efficacy and trust. Journal of Financial Services Marketing, 20(2), 145-157.

Arenas, G., Peral, P., \& Ramón, J. M. (2015). Elderly and internet banking: An application of UTAUT2. Journal of Internet Banking \& Commerce, 20(1), 1-23.

Bộ Nội Vụ. (2019). Báo cáo số 6466/BC-BNV ngày 24/12/2019 của Bộ Nội vụ về tình hình thưc hiện công tác cải cách hành chính năm 2019 [Report No. 6466/BC-BNV dated December 24, 2019 of the Ministry of Home Affairs of the Socialist Republic of Vietnam on the implementation of administrative reform in 2019]. Truy cập ngày 24/12/2019 tại https://moha.gov.vn/danh-muc/bao-cao-so-6466-bc-bnv-ngay-24-12-2019-cua-bo-noi-vuve-tinh-hinh-thuc-hien-cong-tac-cai-cach-hanh-chinh-nam-2019-41443.html

Bộ Y Tế. (2020). Ministry of health portal. Truy cập ngày 11/03/2020 tại https://moh.gov.vn/tintong-hop/-/asset_publisher/k206Q9qkZOqn/content/thu-tuong-chi-thi-ay-manh-phongchong-dich-covid-19-trong-tinh-hinh-moi 
Chan, S. C. (2004). Understanding internet banking adoption and use behavior: A Hong Kong perspective. Journal of Global Information Management, 12(3), 21-43.

Cohen, J. (2013). Statistical power analysis for the behavioral sciences. New York, NY: Academic Press.

Davis, F. D. (1985). A technology acceptance model for empirically testing new end-user information systems: Theory and results. (Doctoral dissertation, Massachusetts Institute of Technology, Cambridge, MA). Truy cập ngày 30/05/2020 tại https://www.researchgate.net/publication/35465050_A_Technology_Acceptance_Model_f or_Empirically_Testing_New_End-User_Information_Systems

Davis, F. D. (1993). User acceptance of information technology: System characteristics, user perceptions and behavioral impacts. International Journal of Man-machine Studies, 38(3), 475-487.

Davis, F. D., Bagozzi, R. P., \& Warshaw, P. R. (1989). Perceived usefulness, perceived ease of use, and user acceptance of information technology. MIS Quarterly, 13(3), 319-340.

Doll, W. J., Hendrickson, A., \& Deng, X. (1998). Using Davis's perceived usefulness and ease of use instruments for decision making: A confirmatory and multigroup invariance analysis. Decision Science, 29(4), 839-870.

Gefen, D., \& Straub, D. (2000). The relative importance of perceived ease of use in IS adoption: A study of e-commerce adoption. Journal of The Association for Information Systems, 1(8), 1-30.

Geisser, S. (1974). A predictive approach to the random effect model. Biometrika, 61(1), 101-107.

Gia Huy (2021). Chính phủ số tại Việt Nam sẽ được hình thành vào năm 2025 [Digital government in Vietnam will be formed by 2025]. Truy cập ngày 10/03/2021 tại Văn phòng Chính phủ (Government Office) website: http://vpcp.chinhphu.vn/Home/Chinh-phu-so-taiViet-Nam-se-duoc-hinh-thanh-vao-nam-2025/20213/29655.vgp

Gorsuch, R. L. (1974). Factor analysis. Philadelphia, Pennsylvania: W.B. Saunders Co.

Hair, J. F., Black, W. C., Babin, B. J., \& Anderson, R. E. (2009). Multivariate data analysis. Hoboken, NJ: Prentice Hall.

Hair, J. F., Hollingsworth, C. L., Randolph, A. B., \& Chong, A. L. (2017). An updated and expanded assessment of PLS-SEM in information systems research. Industrial Management \& Data Systems, 117(3), 442-458.

Hair, J. F., Hult, G. M., Ringle, C., \& Sarstedt, M. (2016). A primer on partial least squares structural equation modeling (PLS-SEM). Thousand Oaks, CA: Sage Publications.

Hair, J. F., Risher, J. J., Sarstedt, M., \& Ringle, C. M. (2019). When to use and how to report the results of PLS-SEM. European Business Review, 31(1), 2-24.

Hidayanto, A. N., \& Ekawati, R. K. (2010). The influence of antecedent factors of IS/IT utilization towards organizational performance: A case study of IAIN Raden Fatah Palembang. Proceeding of the 3rd International Conference on Information and Communication Technology for the Moslem World (ICT4M), 40-47. doi:10.1109/ICT4M.2010.5971937

Hill, T., Smith, N. D., \& Mann, M. F. (1987). Role of efficacy expectations in predicting the decision to use advanced technologies: The case of computers. Journal of Applied Psychology, 72(2), 307-313.

Hsu, C. L., \& Lu, H. P. (2004). Why do people play on-line games? An extended TAM with social influences and flow experience. Information \& Management, 41(7), 853-868. 
Im, I., Hong, S., \& Kang, M. S. (2011). An international comparison of technology adoption: Testing the UTAUT model. Information \& Management, 48(1), 1-8.

Israel, G. D. (1992). Determining sample size. Truy cập ngày 11/03/2021 tại https://www.psycholosphere.com/Determining\%20sample\%20size\%20by\%20Glen\%20Israel.pdf

Kardes, F., \& Cronley, M. (2015). Consumer behavior. Stamford, CT: Cengage Learning.

Khan, M. A. (2007). Consumer behaviour and advertising management. Daryaganj, New Delhi: New Age International.

Kim, H. W., \& Kankanhalli, A. (2009). Investigating user resistance to information systems implementation: A status quo bias perspective. MIS Quarterly, 33(3), 567-582.

Kim, S. S., \& Malhotra, N. K. (2005). A longitudinal model of continued IS use: An integrative view of four mechanisms underlying postadoption phenomena. Management Science, 51(5), 741-755.

Kim, S. S., Malhotra, N. K., \& Narasimhan, S. (2005). Research note - two competing perspectives on automatic use: A theoretical and empirical comparison. Information Systems Research, 16(4), 418-432.

Kline, P. (2014). An easy guide to factor analysis. Abingdon, Oxfordshire: Routledge.

Lederer, A. L., Maupin, D. J., Sena, M. P., \& Zhuang, Y. (2000). The technology acceptance model and the World Wide Web. Decision Support Systems, 29(3), 269-282.

Likert, R. (1932). A technique for the measurement of attitudes. Archives of Psychology, 22(140), 1-55.

Limayem, M., Hirt, S. G., \& Cheung, C. M. (2007). How habit limits the predictive power of intention: The case of information systems continuance. MIS Quarterly, 31(4), 705-737.

Lippert, S. K., \& Davis, M. (2006). A conceptual model integrating trust into planned change activities to enhance technology adoption behavior. Journal of Information Science, 32(5), 434-448.

Moon, J. W., \& Kim, Y. G. (2001). Extending the TAM for a World-Wide-Web context. Information and Management, 38(4), 217-230.

Moore, G. C., \& Benbasat, I. (1991). Development of an instrument to measure the perceptions of adopting an information technology innovation. Information Systems Research, 2(3), 192-222.

Nguyen, T. D., Nguyen, D. T., \& Cao, H. T. (2014). Acceptance and use of information system: E-learning based on cloud computing in Vietnam. In Information and Communication Technology-EurAsia Conference (pp.139-149). Berlin, Heidelberg: Springer.

Nunnally, J. C., \& Bernstein, I. H. (1994). Psychometric theory. New York, NY: McGraw-Hill.

Peng, D. X., \& Lai, F. (2012). Using partial least squares in operations management research: A practical guideline and summary of past research. Journal of Operations Management, $30(6), 467-480$.

Pham, T., Dang, L., \& Le, T. (2020). Factors affecting teachers' behavioral intention of using information technology in lecturing-economic universities. Management Science Letters, 10(11), 2665-2672.

Raman, A., \& Don, Y. (2013). Preservice teachers' acceptance of learning management software: An application of the UTAUT2 model. International Education Studies, 6(7), 157-164. 
Ringle, C. M., Sarstedt, M., \& Straub, D. W. (2012). A critical look at the use of PLS-SEM in MIS. MIS Quarterly, 36(1), 3-14.

Ringle, C. M., Sarstedt, M., Mitchell, R., \& Gudergan, S. P. (2020). Partial least squares structural equation modeling in HRM research. The International Journal of Human Resource Management, 31(12), 1617-1643.

Segars, A. H., \& Grover, V. (1993). Re-examining perceived ease of use and usefulness: A confirmatory factors analysis. MIS Quarterly, 17(4), 517-526.

Sharma, D. (2013). Resistance to Human Resource Information Systems (HRIS)-problem recognition, diagnosis, and positive intervention: A study on employee behavior and change management. Indian Journal of Applied Research, 3(1), 99-104.

Sosik, J. J., Kahai, S. S., \& Piovoso, M. J. (2009). Silver bullet or voodoo statistics? A primer for using the partial least squares data analytic technique in group and organization research. Group \& Organization Management, 34(1), 5-36.

Stone, M. (1974). Cross validatory choice and assessment of statistical predictions. Journal of the Royal Statistical Society: Series B (Methodological), 36(2), 111-133.

Tapanainen, T., Dao, K. T., Nguyen, H. T., \& Nguyen, H. T. T. (2019). 4G adoption: A survey of Vietnam market. Journal of Information Technology Applications \& Management, 26(1), 1-19.

Teo, T. (2011). Factors influencing teachers' intention to use technology: Model development and test. Computers \& Education, 57(4), 2432-2440.

Thompson, R. L., Higgins, C. A., \& Howell, J. M. (1991). Personal computing: Toward a conceptual model of utilization. MIS Quarterly, 15(1), 125-143.

Venkatesh, V., \& Davis, F. D. (1996). A model of the antecedents of perceived ease of use: Development and test. Decision Sciences, 27(3), 451-481.

Venkatesh, V., \& Davis, F. D. (2000). A theoretical extension of the technology acceptance model: Four longitudinal field studies. Management Science, 46(2), 186-204.

Venkatesh, V., \& Zhang, X. (2010). Unified theory of acceptance and use of technology: US vs. China. Journal of Global Information Technology Management, 13(1), 5-27.

Venkatesh, V., Morris, M. G., Davis, G. B., \& Davis, F. D. (2003). User acceptance of information technology: Toward a unified view. MIS Quarterly, 27(3), 425-478.

Venkatesh, V., Thong, J. Y., \& Xu, X. (2012). Consumer acceptance and use of information technology: Extending the unified theory of acceptance and use of technology. MIS Quarterly, 36(1), 157-178.

Wang, M. H. (2016). Factors influencing usage of e-learning systems in Taiwan's public sector: Applying the utaut model. Advances in Management and Applied Economics, 6(6), 63-82.

Warshaw, P. R., \& Davis, F. D. (1985). Disentangling behavioral intention and behavioral expectation. Journal of Experimental Social Psychology, 21(3), 213-228.

Wong, K. T., Teo, T., \& Russo, S. (2013). Interactive whiteboard acceptance: Applicability of the UTAUT model to student teachers. The Asia-Pacific Education Researcher, 22(1), 1-10.

\footnotetext{
Creative Commons Attribution-NonCommercial 4.0 International License.
} 